\begin{tabular}{|c|l|}
\hline III Simpósio Paranaense de Modelagem, & Artigo: 31 \\
$\begin{array}{c}\text { Simulaçăoe Controle de Processos } \\
\text { ISSN : 1984-7521 }\end{array}$ & Páginas: $230-235$
\end{tabular}

\title{
MÉTODO DE CALIBRAÇÃO DE SENSORES DE TEMPERATURA UTILIZANDO ARDUINO - LABENGE - UFPR
}

\author{
Alisson L. Daga ${ }^{1}$ e Marcelo K. Lenzi ${ }^{2}$ \\ ${ }^{1}$ Universidade Federal do Paraná, Departamento de Engenharia Química - e-mail: alisson.daga@ufpr.br \\ ${ }^{2}$ Universidade Federal do Paraná, Programa de Pós-Graduação em Engenharia Química - e-mail: lenzi@ufpr.br
}

\begin{abstract}
Resumo. Utilizando-se o ARDUINO foi possível montar um módulo experimental para a instrução de alunos em práticas de calibração. O experimento consiste em coletar dados experimentais de temperatura cujos valores são considerados verdadeiros a partir de sensores de baixo custo aplicados ao ARDUINO. Assim, o aluno pode ajustar uma reta de calibração após ter de fato calibrado o instrumento. Em particular, foram utilizados 6 dados experimentais de temperatura, sendo que por meio da metodologia proposta foi possível confeccionar um módulo didático de calibração. O aluno interage com módulo por meio da resistência do TRIMPOT e é estimulado a relembrar conceitos básicos de estatística e incertezas para conseguir obter o valor real da temperatura de uma amostra com a respectiva incerteza.
\end{abstract}

Palavras-chave: Tecnologia na Educação, Arduino, Software Livre, Calibração.

\section{INTRODUÇÃO}

Existem diferentes formas de compreender a tecnologia, tais como, tornar o trabalho mais leve, uma locomoção e comunicação mais fácil ou simplesmente tornar a vida mais divertida e satisfatória. E neste contexto a tecnologia não é algo novo, mas na verdade é algo tão velho quanto o próprio homem. Pode-se classificar as tecnologias em as que estendem a força física do homem, outras que permitem mover pelo espaço mais rapidamente e as que amplificam os poderes sensoriais do homem, a última sem dúvida é altamente relevante para a educação (Chaves, 1999).

As tecnologias que amplificam os poderes sensoriais do homem são relativamente recentes, comparando com outras, e mais recente ainda são as que potencializam a capacidade mental do homem, que foi o desenvolvimento do computador digital. A difusão da computação digital, iniciouse na década de 70, a sua popularização e a sua interligação em redes na década de 80, e nos anos 90 com a explosão da internet (Lemos, 2002). Com a popularização da informática, a necessidade de softwares seguiu na mesma tendência. Podemos descrever os softwares como um conjunto de componentes lógicos que são necessários para realizar um trabalho, que ao contrário do hardware, compõe os componentes físicos.

$\mathrm{Na}$ linha do tempo da evolução da computação, em que o conceito da cooperação foi sempre presente entre as comunidades de programadores no desenvolvimento de softwares, no início dos anos 80 quase todos os programas existentes passaram a ser privativos, e o conceito de propriedade invadiu a área de tecnologia. Neste contexto surgiu a figura do software livre, introduzido por Stallman na qual iniciou um movimento para produzir um sistema operacional e programas que empodera o usuário, resguardando as liberdades que os programadores conheciam antes da imposição das restrições empresariais e rompe com a submissão dos usuários à um fornecedor de software (Evangelista, 2004).

Após anos de desenvolvimento e miniaturização de componentes eletrônicos, na Itália foi desenvolvido uma plataforma de prototipação de hardware pelo italiano Massimo Banzi, que em 2005 iniciou seus estudos para resolver uma dificuldade que acometia o cenário acadêmico do Instituto de Design de Ivrea (Arduino, 2012). Os estudos de Banzi contribuiram significativamente para o desenvolvimento de uma família de microcontroladores destinada à indivíduos com pouca ou nenhuma familiaridade com eletrônica e programação, assim facilitando a integração de componentes eletrônicos com comportamentos singulares nos protótipos desenvolvidos por alunos. 
O Arduino é um computador miniaturizado em que você pode obter resultado ou ações a partir de leituras de entradas, fornecidas através de componentes externos, que tratadas através de um programa específico, lhe retornará os resultados desejados (McRoberts, 2011).

A tecnologia livre é uma aliada nas práticas de ensino, onde proporciona facilidade de utilização com o público que não têm familiaridade com eletrônica e programação, e atrativo na comunidade educacional, devido ao custo deixar de ser um problema (Soares, 2016).

A aplicação dos conceitos do Arduino, é utilizada na metodologia de ensino sobre a calibração de instrumentos de laboratório, em que o conjunto de hardware e software oferece ao aluno a efetiva experiência em calibrar um instrumento em relação a um valor de referência, e testar utilizando de métodos estatísticos o desempenho de sua calibração.

\section{MATERIAIS E MÉTODOS}

O estudo da utilização de hardware livre e software livre, para aplicação na metodologia de ensino de calibração de instrumentos, é constituído dos seguintes elementos.

- Arduino UNO (Figura 1a)

O hardware livre Arduino UNO, é uma placa de desenvolvimento microcontrolada baseada no ATmega328P, ela possui 14 pinos de entrada/saída digital, 6 entradas analógicas, um cristal oscilador de $16 \mathrm{MHz}$, uma conexão USB, uma entrada para alimentação, um cabeçalho ICP e um botão reset.

- Banho Termostático (Figura 1b)

Banho Ultratermostático com funções de aquecimento e refrigeração, com faixa de operação de $-20^{\circ} \mathrm{C}$ a $200^{\circ} \mathrm{C}$, modelo UNITEMP.

- Tela OLED 0,96" I2C (Figura 1c)

Display OLED azul, com 0,96 " e protocolo de comunicação I2C controlado pelo chip

SSD1306, têm luz própria e não necessita de backlight, o que intensifica o contraste com pouca energia.

- TRIMPOT (Figura 1d)

É um potenciômetro ajustável em miniatura, de resitência $100 \mathrm{~K} \Omega$, são comuns em sistemas de precisão, e no nosso caso em estudo, é o componente que fará o ajuste da temperatura medida em comparação com a temperatura padrão.

- Sensor de temperatura DS18B20 (Figura 1e)

O sensor de temperatura DS18B20, é um sensor digital de temperatura que pode ser alimentado com tensões DC entre $3.0 \mathrm{~V}$ e $5.5 \mathrm{~V}$, mede temperaturas na faixa de $-55^{\circ} \mathrm{C}$ e $+125^{\circ} \mathrm{C}$, não necessita de componentes externos, resolução ajustável entre 9 e 12 bits, à prova da água, umidade e ferrugem, utiliza o protocolo 1-Wire para leitura.

Pode-se observar o conjunto em funcionamento, conforme apresentado na Figura 1(f).

O procedimento experimental consiste na hipótese inicial de a temperatura do banho termostático ser considerada verdadeira, ou seja, a temperatura real. Desta forma, a temperatura do banho deve ser definida conforme os valores da Tabela 1 . Por ocasião da $1^{\mathrm{a}}$. medição, ou seja, $20,0^{\circ} \mathrm{C}$, o sistema deve ser calibrado por meio da manipulação da resistência do TRIMPOT, alterando-se a quantidade de voltas do parafuso, indicado na Figura 1(f). Desta forma, o aluno está calibrando o instrumento até que a leitura do módulo coincida com a indicação de temperatura do banho. Este procedimento é realizado apenas por ocasião da $1^{\mathrm{a}}$. medição, com o objetivo de verificar se o sistema se mantém calibrado ao longo do experimento. Assim, após o sistema estar calibrado, podem ser obtidas as demais medições de forma a preencher a Tabela 1. 
Tabela 1 - Valores de temperatura

\begin{tabular}{|c|c|c|c|c|c|c|}
\hline $\mathrm{n}$ (medida) & 1 & 2 & 3 & 4 & 5 & 6 \\
\hline $\mathrm{T}^{\text {real }}-\left[{ }^{\circ} \mathrm{C}\right]$ & 20,0 & 25,0 & 30,0 & 35,0 & 40,0 & 45,0 \\
\hline $\mathrm{T}^{\text {módulo }}-\left[{ }^{\circ} \mathrm{C}\right]$ & & & & & & \\
\hline
\end{tabular}
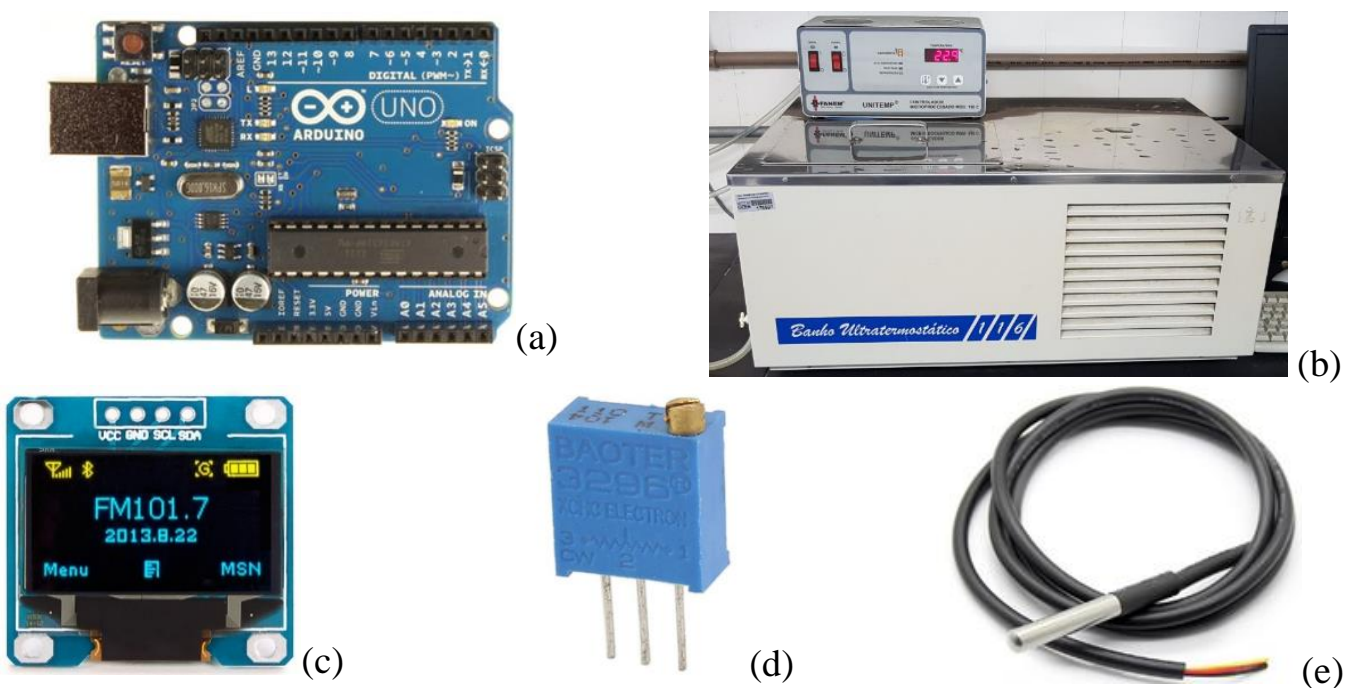

(d)

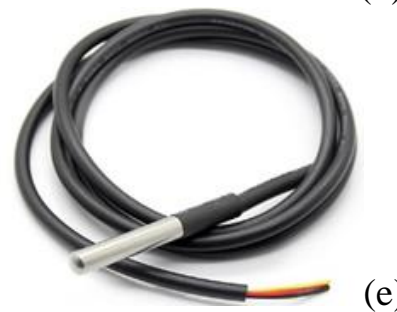

(e)

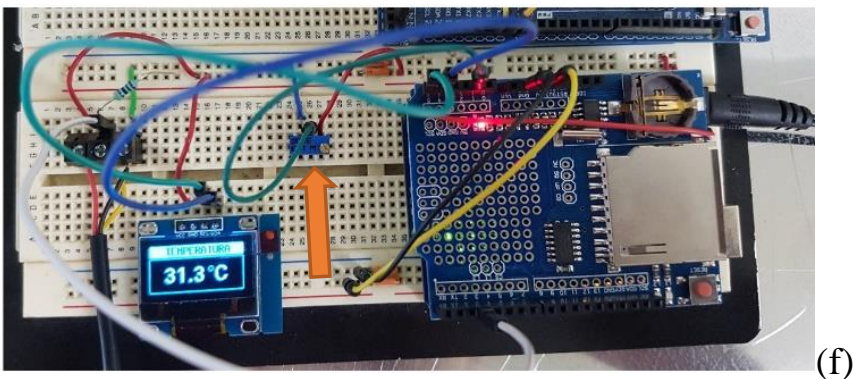

Figura 1 - Materiais utilizados. (a) Arduino UNO; (b) Sensor DS18B20; (c) Display OLED 0,96" I2C; (d) TRIMPOT $10 \mathrm{k} \Omega$; (e) Componentes integrados em protoboard

Após a coleta de dados, uma reta de calibração deve ser ajustada, sendo determinados dos coeficientes angular e linear, bem como a respectiva incerteza. Além de relembrar os conceitos de estatística, o aluno irá utilizar esta informação para prever o valor da temperatura de uma amostra independente de validação, vem como a incerteza desta predição. $\mathrm{O}$ equacionamento a ser utilizado é apresentado a seguir, sendo referenciado por SCHWAAB \& PINTO (2007)

Portanto, a partir dos dados experimentais, será ajustada a seguinte reta $T_{i}^{\text {real-predita }}=a \cdot T_{i}^{\text {módulo-exp }}+b$

Será utilizada como função objetivo a minimização das distâncias quadráticas entre dados experimentais e predições da reta, dada pela equação a seguir,

$$
F=\sum_{i=1}^{N E}\left(T_{i}^{\text {real-exp }}-T_{i}^{\text {real-predita }}\right)^{2}=\sum_{i=1}^{N E}\left(T_{i}^{\text {real-exp }}-\left(a \cdot T_{i}^{\text {módulo-exp }}+b\right)\right)^{2}
$$

Onde NE é o número de pontos experimentais, neste estudo este valor é 6, conforme a Tabela 1. 
Como a reta é um modelo linear nos parâmetros, o problema de estimação de parâmetros possui solução única e analítica dada pela Eq. (3), sob a forma matricial. Esta pode ser obtida a partir da solução do sistema de equações obtido a partir das derivadas de F em relação aos parâmetros "a" $\mathrm{e}$ "b" igualadas a zero.

$$
\begin{aligned}
& \frac{\partial F}{\partial a}=0 \rightarrow 2 \cdot\left[\sum_{i=1}^{N E}\left(y_{i}^{\text {exp }}-\left(a \cdot T_{i}^{\text {módulo-exp }}+b\right)\right)\right] \cdot\left(-T_{i}^{\text {real-exp }}\right)=0 \\
& \frac{\partial F}{\partial b}=0 \rightarrow 2 \cdot\left[\sum_{i=1}^{N E}\left(y_{i}^{\text {exp }}-\left(a \cdot T_{i}^{\text {módulo-exp }}+b\right)\right)\right] \cdot(-1)=0 \\
& \underbrace{\left[\begin{array}{ll}
\sum_{i=1}^{N E}\left(T_{i}^{\text {módulo-exp }}\right)^{2} & \sum_{i=1}^{N E}\left(T_{i}^{\text {módulo-exp }}\right) \\
\sum_{i=1}^{N E}\left(T_{i}^{\text {módulo-exp }}\right) & \sum_{i=1}^{N E} 1
\end{array}\right]}_{\underline{\underline{A}}} \cdot\left[\begin{array}{l}
a \\
b
\end{array}\right]=\underbrace{\left[\begin{array}{l}
\sum_{i=1}^{N E}\left(T_{i}^{\text {real-exp }} \cdot T_{i}^{\text {módulo-exp }}\right) \\
\sum_{i=1}^{N E}\left(T_{i}^{\text {real-exp }}\right)
\end{array}\right]}_{\underline{m}} \rightarrow \underline{A} \cdot \underline{k}=\underline{m} \rightarrow \underline{k}=(\underline{A})^{-1} \cdot \underline{m}
\end{aligned}
$$

A variância experimental pode ser aproximada pela Eq. (5).

$$
S_{\text {exp }}^{2}=\frac{F}{N E-N P}
$$

onde NP é o número de parâmetros do modelo, neste estudo, a reta possui 2 parâmetros.

Com isso, a variância dos coeficientes da reta é dada pelos elementos da diagonal principal da matriz da Eq. (6), e na diagonal secundária está a correlação paramétrica

$$
\underline{M}=s_{\exp }^{2} \cdot(\underline{\underline{A}})^{-1}=\left[\begin{array}{cc}
s_{a}^{2} & s_{a-b}^{2} \\
s_{a-b}^{2} & s_{b}^{2}
\end{array}\right]
$$

Assim, a partir de técnicas de propagação de erro, a incerteza na predição de um valor de temperatura não utilizado na estimação de parâmetros é dada pela incerteza do próprio modelo somada à incerteza experimental, conforme a Eq. (8).

$$
\begin{aligned}
& \mathrm{s}_{\text {modelo }}^{2}=\left(\frac{\partial T_{i}^{\text {real-predita }}}{\partial a}\right)^{2} \cdot s_{a}^{2}+\left(\frac{\partial T_{i}^{\text {real-predita }}}{\partial b}\right)^{2} \cdot s_{b}^{2}+2 \cdot\left(\frac{\partial T_{i}^{\text {real-predita }}}{\partial a}\right) \cdot\left(\frac{\partial T_{i}^{\text {real-predita }}}{\partial b}\right) \cdot s_{a-b}^{2} \\
& S_{\text {modelo }}^{2}=\left(T_{i}^{\text {módulo-exp }}\right)^{2} \cdot s_{a}^{2}+(1)^{2} \cdot s_{b}^{2}+2 \cdot\left(T_{i}^{\text {módulo-exp }}\right) \cdot(1) \cdot s_{a-b}^{2} \\
& S_{\text {final }}^{2}=S_{\text {exp }}^{2}+S_{\text {modelo }}^{2}
\end{aligned}
$$

\section{RESULTADOS E DISCUSSÃO}

A Tabela 1 apresenta os dados experimentais obtidos a partir do banho termostático $\left(\mathrm{T}^{\text {real }}\right)$ após a calibração do módulo, por meio da manipulação da resistência do TRIMPOT indicado na Figura 1(f).

Tabela 1 - Valores de temperatura

\begin{tabular}{|c|c|c|c|c|c|c|}
\hline $\mathrm{n}$ (medida) & 1 & 2 & 3 & 4 & 5 & 6 \\
\hline $\mathrm{T}^{\text {real }}-\left[{ }^{\circ} \mathrm{C}\right]$ & 20,0 & 25,0 & 30,0 & 35,0 & 40,0 & 45,0 \\
\hline $\mathrm{T}^{\text {módulo }}-\left[{ }^{\circ} \mathrm{C}\right]$ & 20,0 & 24,8 & 29,9 & 34,7 & 39,6 & 44,5 \\
\hline
\end{tabular}


Utilizando-se o equacionamento descrito na seção anterior, foi possível obter os resultados apresentados na Tabela 2. A Figura 2 ilustra graficamente os resultados experimentais e a reta de calibração, indicando um excelente ajuste experimental, corroborado pelo pequeno valor da função objetivo, F. Além disso, salienta-se que ambos os parâmetros estimados são estatisticamente significativos tendo em vista que as respectivas variâncias levam à desvios padrões menores que o próprio valor do parâmetro.

Tabela 2 - Estimação de parâmetros

\begin{tabular}{|c|c|}
\hline Parâmetro "a" & 1,02 \\
\hline Variância de "a" - $\mathrm{s}_{a}^{2}$ & $1,2 \cdot 10^{-5}$ \\
\hline Parâmetro "b" & $-0,37$ \\
\hline Variância de "b" - $\mathrm{s}_{b}^{2}$ & $1,3 \cdot 10^{-2}$ \\
\hline $\begin{array}{c}\text { Covariância "a-b" - } \\
\mathrm{s}_{a-b}^{2}\end{array}$ & $-3,9 \cdot 10^{-4}$ \\
\hline $\mathrm{F}$ & 0,0202 \\
\hline $\mathrm{s}_{\text {exp }}^{2}$ & 0,00505 \\
\hline $\mathrm{s}_{\text {modelo }}^{2}$ & {$\left[\left(\mathrm{~T}_{\mathrm{i}}^{\text {módulo-exp }}\right)^{2} \cdot\left(1,2 \cdot 10^{-5}\right)\right]+\left(1,3 \cdot 10^{-2}\right)+\left[2 \cdot\left(\mathrm{T}_{i}^{\text {módulo-exp }}\right) \cdot\left(-3,9 \cdot 10^{-4}\right)\right]$} \\
\hline
\end{tabular}

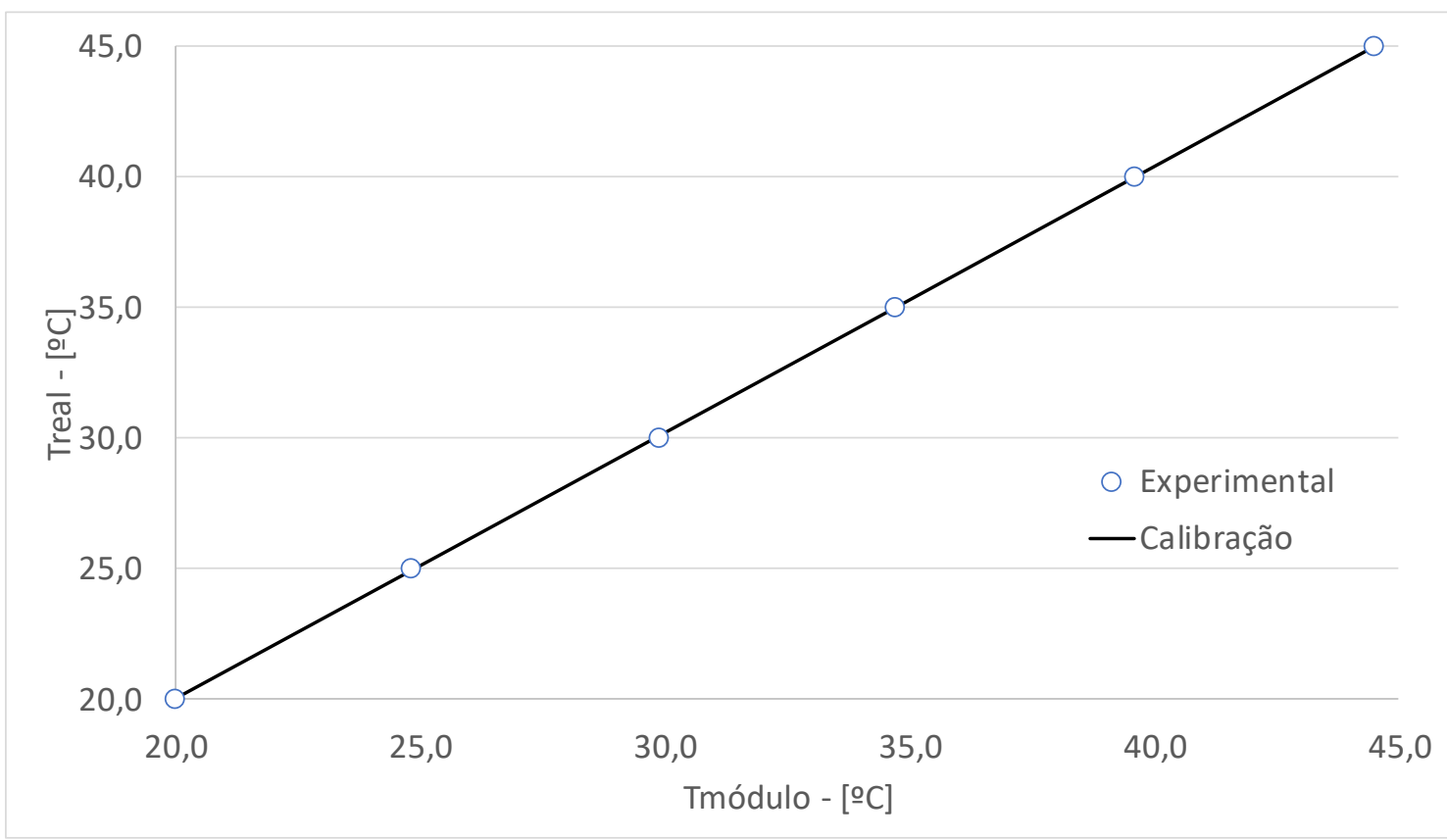

Figura 2 - Valores experimentais versus preditos pelo modelo

Para fins de validação, foi elaborada uma amostra teste independente. Assim, misturando-se água quente com água fria, obteve-se uma amostra cujo valor de temperatura medido pelo módulo experimental foi de $33,7^{\circ} \mathrm{C}$. Mas, deve-se ter em mente que este não é o valor real da temperatura, o qual de fato pode ser obtido pela Eq. (1) com os parâmetros "a" e "b" estimados e apresentados na Tabela 2, resultando em um valor de

$$
T_{i}^{\text {real-predita }}=1,02 \cdot T_{i}^{\text {módulo-exp }}-0,37=1,02 \cdot(33,7)-0,37=34,0^{\circ} \mathrm{C}
$$


Além disso, a variância desta predição é dada por

$$
s_{\text {final }}=\sqrt{s_{\text {exp }}^{2}+s_{\text {modelo }}^{2}}=\sqrt{(0,00505)+(0,00087)}=\sqrt{0,00592}=0,077 \simeq 0,1
$$

onde

$$
\begin{array}{cc}
S_{\text {exp }}^{2} & 0,00505 \\
S_{\text {modelo }}^{2} & {\left[\left(T_{i}^{\text {módulo-exp }}\right)^{2} \cdot\left(1,2 \cdot 10^{-5}\right)\right]+\left(1,3 \cdot 10^{-2}\right)+\left[2 \cdot\left(T_{i}^{\text {módulo-exp }}\right) \cdot\left(-3,9 \cdot 10^{-4}\right)\right]} \\
& {\left[(33,7)^{2} \cdot\left(1,2 \cdot 10^{-5}\right)\right]+\left(1,3 \cdot 10^{-2}\right)+\left[2 \cdot(33,7) \cdot\left(-3,9 \cdot 10^{-4}\right)\right]=0,00087}
\end{array}
$$

Desta forma, a temperatura da amostra pode ser representada por $34,0 \pm 0,1^{\circ} \mathrm{C}$.

\section{CONCLUSÃO}

Por meio da metodologia proposta foi possível confeccionar um módulo didático de calibração de temperatura. $\mathrm{O}$ aluno interage com módulo por meio da resistência do TRIMPOT e é estimulado a relembrar conceitos básicos de estatística e incertezas para conseguir obter o valor real da temperatura de uma amostra com a respectiva incerteza.

\section{AGRADECIMENTOS}

Os autores agradecem à UFPR e a CAPES pelo auxílio financeiro.

\section{REFERENCIAS}

ARDUINO. Arduino. 2012. Disponível em: http://www.arduino.cc/. Acesso em: 26/02/2018.

CHAVES, E. Conceitos Básicos: Educação a Distância. EdutecNet: Rede de Tecnologia na Educação, 1999. Disponível em: http://www.edutecnet.com.br/. Acesso em: 26/02/2018.

EVANGELISTA, R. Livre acima de tudo, 2004. Disponível em: http://www.dicas1.com.br/zonadecombate/. Acesso em: 26/02/2018.

LEMOS, A. Cibercultura. Tecnologia e Vida Social na Cultura Contemporânea. Sulina: Porto Alegre, 2002.

MCROBERTS, M. Arduino Básico. Editora NOVATEC: São Paulo. 2011.

SCHWAAB, M.; PINTO, J.C. Análise de Dados Experimentais. I. Fundamentos de Estatística e Estimação de Parâmetros. Editora e-papers: Rio de Janeiro, 2007.

SOARES, Renira Carla. Utilização da plataforma de prototipação de Hardware Arduino como apoio à aprendizagem de conceitos do componente curricular de programação. 2016. Relatório Interno. 